\title{
The ups and downs of negative (and positive) selection of $B$ cells
}

\author{
Jean-Claude Weill and Claude-Agnès Reynaud \\ Institut Necker-Enfants Malades (INEM), INSERM U1151-CNRS UMR 8253, Université Paris Descartes, Faculté de Médecine, Sorbonne Paris Cité, Paris, France.
}

\begin{abstract}
Central and peripheral tolerance checkpoints are in place to remove autoreactive B cell populations and prevent the development of autoimmunity. In this issue of the $J C I$, Pala and colleagues reveal that individuals with the X-linked immunodeficiency Wiskott-Aldrich syndrome (WAS) have opposite alterations at central and peripheral B cell checkpoints: a more stringent selection for central tolerance, resulting in reduced numbers of autoreactive cells at the emergent immature B cell stage, and a relaxed selection for peripheral tolerance, resulting in an increased frequency of autoreactive cells in the mature naive B cell compartment. Moreover, reinstatement of the WAS gene in these patients restored both B cell tolerance checkpoints. These results suggest that, in a normal situation, mature naive $B$ cells undergo a positive selection step driven by selfantigens, kept in control by Tregs.
\end{abstract}

Central and peripheral tolerance mechanisms keep autoreactive lymphocytes in check

The clonal selection theory of adaptive immunity remains the conceptual framework of modern immunology and states that the immune system develops to recognize external antigens while avoiding self-antigens (1). If clonal selection is not properly maintained, pathological autoimmune consequences may arise. Lymphocytes are composed of $\mathrm{T}$ and $\mathrm{B}$ cell populations that are generated in two different organs (thymus and BM, respectively) that independently take care of self/nonself discrimination. In the thymus, $\mathrm{T}$ cells are educated to paradoxically recognize but also avoid self-recognition through successive positive and negative selection. The final outcome of this process is the emigration of a pool of CD4 and CD8 naive T cells, as well as a population of Tregs to the periphery. Less than $10 \%$ of $\mathrm{T}$ lymphocyte precursors that pass through the thymus make it to the periphery, as most die of neglect at the positive selection step. These intrathymic selection stages have been rather well defined, even though the MHC-associated peptides involved in positive selection have not yet been fully characterized (2). Elegant transgenic models have shown that, for B cells, the purging of potentially dangerous anti-self B cell clones (negative selection) not only takes place in BM during their generation but also at the periphery after $B$ cells have reached their mature state. Removal of autoreactive B cells involves modification of the specificity of the $\mathrm{B}$ cell receptor (BCR) through editing of its $\mathrm{V}_{\mathrm{L}}$-encoding gene or the elimination of the $\mathrm{B}$ cell by clonal deletion. Anergy has also been described as a third mechanism for eradication of errant $\mathrm{B}$ cells and implies a functional inactivation of their signaling capacity $(3,4)$.

More recently, cloning and expression of the BCR from single human B cells has allowed quantification of the extent of negative selection taking place in $\mathrm{BM}$ and in the periphery at both the immature and mature B cell differentiation stages (5). Strikingly,

Related Article: p. 3941

Conflict of interest: Jean-Claude Weill is president of the scientific council of the Mérieux Research Grants.

Reference information: J Clin Invest. 2015;125(10):3748-3750. doi:10.1172/JCI84009.

the results have shown that around $70 \%$ of precursor $\mathrm{B}$ cells produced in the BM display autoreactive specificities against self-antigens, such as cytoplasmic and nuclear cellular constituents, and are polyspecific, with the ability to bind different antigenic structures. After B cells have passed through the two tolerance checkpoints in BM and at the periphery, around $20 \%$ of the remaining $B$ cells recognize self-structures (Figure 1). It has been argued that the major function of these anti-self antibodies is the active clearance of tissue and cell debris constantly generated by dying cells (6). Previous work by Eric Meffre and colleagues has dissected in detail the molecular signals involved in these two selection checkpoints by studying patients who display primary immunodeficiency diseases (7). The conclusion from these studies was that central tolerance is mainly controlled by intrinsic B cell signals involving the BCR and TLRs, while peripheral tolerance is controlled by extrinsic signals that are essentially produced by Tregs.

\section{Wiskott-Aldrich syndrome patients provide new insight into B cell tolerance checkpoints}

In this issue, Pala and colleagues add another twist to the story by studying patients with Wiskott-Aldrich syndrome (WAS), an X-linked primary immunodeficiency that is characterized by both cellular and humoral defects, along with an increased risk of developing autoimmunity and lymphomas (8). The WAS gene-encoded product is a key regulator of actin polymerization, and several studies in both murine models and individuals with WAS have shown that WAS deficiency induces alterations in the Treg and effector $\mathrm{T}$ cell compartment, as well as dysfunctional removal of autoreactive B cells (9). Pala et al. now show that there is, rather unexpectedly, a decreased frequency of autoreactive new emigrants/ transitional B cells coming out of the $\mathrm{BM}$ in WAS patients, which suggests that 


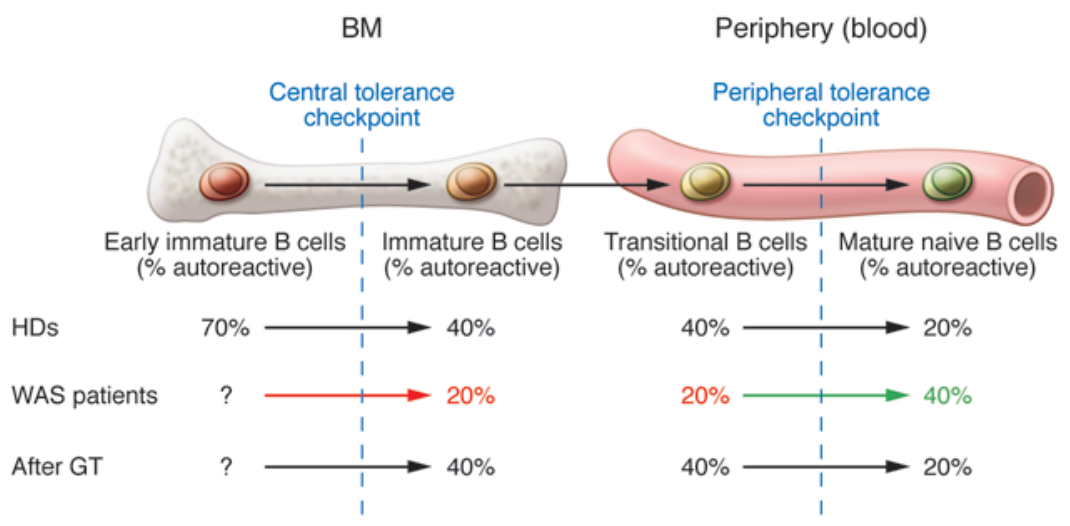

Figure 1. GT corrects abnormal B cell tolerance checkpoints in patients with WAS. Autoreactivity, as measured through the Hep-2 cell binding assay, is controlled at two steps: in the BM at the transition from pre-B to immature $B$ cell stage (central tolerance) and at the periphery (evaluated in blood) at the transitional/immature to mature B cell stage (peripheral tolerance). In healthy donors (HDs), $70 \%$ of pre-B cells are autoreactive, with the central tolerance checkpoint reducing the proportion of autoreactive immature B cells to $40 \%$. Following transition to the periphery, peripheral tolerance checkpoints result in autoreactivity in only $20 \%$ of the mature B cell population (5). B cells in WAS patients have an unusual profile with enhanced negative selection at the central tolerance checkpoint and relaxed negative (i.e., increased positive selection) at the peripheral checkpoint. Both anomalies are corrected after GT to restore a functional WAS gene.

these cells are hyperreactive to stimulation through their BCR and thus have a lowered threshold for negative selection, a hypothesis validated for patients' mature B cells (8). Conversely, peripheral tolerance in these patients was impaired, with a surprising reversal in the frequency of autoreactive cells compared with the immediate B cell precursor subset (Figure 1). The enhanced frequency of autoreactive B cells was likely due to the presence of a defective Treg population observed in the absence of the WAS gene.

WAS is among an increasing list of hematopoietic diseases for which gene therapy (GT), through corrected expression of the affected gene in hematopoietic stem cells of the patients, has potential to lead to a durable disease remission (10, 11). Pala and colleagues further demonstrated that such GT in individuals with WAS restores a normal immune system and a normal pattern of autoreactivity among B cells (Figure 1). This group has also reported a similar restoration of tolerance checkpoints in patients with adenosine deaminase deficiency after correction by GT (12). Nevertheless, the effect of GT appears particularly striking in the present case, considering that only partial correction of the lymphoid compartment was achieved at the time points studied (between one and two years after stem cell transfer), with $50 \%-70 \%$ of the lymphoid compartment and only $50 \%$ of B cells expressing the corrected gene (13).

\section{Conclusions and remaining questions}

This study by Pala et al. is the first observation, to our knowledge, of a genetic defect that has opposite consequences on the two tolerance checkpoints for B cells. These results clearly indicate that these two checkpoints must involve different mechanisms and different cells; the results also shed some light on the somehow still mysterious stage of B cell positive selection. Autoreactive B cell clones are abnormally augmented in the periphery of WAS patients, suggesting that positive selection of follicular B cells by self-antigens is taking place, but in normal conditions, this population is maintained at a certain quantitative and qualitative level by Tregs (14). The question remains as to how Tregs exert their control on naive $B$ cells that express an anti-self BCR. They could act by direct suppression (15) or, most likely, through the control of effector $T$ cells, as suggested by the defect in $\mathrm{B}$ cell peripheral tolerance observed in SAP-deficient patients who display a functional Treg population but a $\mathrm{T}$ cell population that is resistant to Tregmediated suppression (16). Such anti-self $\mathrm{T}$ cells therefore escape the thymus and can be activated by self-antigens. In the absence of Tregs, these anti-self $\mathrm{T}$ cells can generate harmful autoreactive clones and also give surviving signals to autoimmune B cells presenting their cognate selfantigens. Paradoxically, as shown in mice, the same anti-self $\mathrm{T}$ cells may also induce FAS-dependent elimination of autoimmune anergic $B$ cells that have migrated to the splenic T cell zone (17).

The general picture of B cell checkpoint tolerance reveals a very subtle equilibrium with a complexity that gives insight into the difficulties encountered when trying to find the etiology of tolerance breakage during an autoimmune process. In conclusion, these data provide, to our knowledge, the first strong experimental evidence that, like $\mathrm{T}$ cells, a large fraction of follicular B cells in the periphery have been positively selected by selfconstituents. As proposed for $\mathrm{T}$ cells, the presence of these same self-antigens in the periphery could allow B cells to constantly check their tonic signaling status.

\section{Acknowledgments}

Jean-Claude Weill and Claude-Agnès Reynaud are supported by an ERC Advanced Grant (Memo-B), the Ligue Nationale contre le Cancer (Equipe labellisée), and the Fondation Princesse Grace de Monaco.

Address correspondence to: Claude-Agnès Reynaud, INEM, INSERM U1151/CNRS UMR8253, Faculté de Médecine Paris Descartes - Site Broussais, Bâtiment Leriche, CS61431, 14 Rue Maria Helena Vieira Da Silva, 75993 Paris Cedex 14, France. Phone: 33.1.72.60.64.48; E-mail: claudeagnes.reynaud@inserm.fr. Or to: JeanClaude Weill, INEM, INSERM U1151/ CNRS UMR8253, Faculté de Médecine Paris Descartes - Site Broussais, Bâtiment Leriche, CS61431, 14 Rue Maria Helena Vieira Da Silva, 75993 Paris Cedex 14, France. Phone: 33.1.72.60.64.47; E-mail: jeanclaude.weill@inserm.fr.

1. Burnet FM. The Clonal Selection Theory of Acquired Immunity. Cambridge, United Kingdom: The University Press; 1959.

2. Klein L, Kyewski B, Allen PM, Hogquist KA. Positive and negative selection of the $\mathrm{T}$ cell repertoire: what thymocytes see (and don't see). Nat Rev Immunol. 2014;14(6):377-391.

3. Ait-Azzouzene D, et al. Tolerance-induced receptor selection: scope, sensitivity, locus specificity, and relationship to lymphocyte-positive 
selection. Immunol Rev. 2004;197:219-230.

4. Miosge LA, Goodnow CC. Genes, pathways and checkpoints in lymphocyte development and homeostasis. Immunol Cell Biol. 2005;83(4):318-335.

5. Wardemann H, Yurasov S, Schaefer A, Young JW, Meffre E, Nussenzweig MC. Predominant autoantibody production by early human B cell precursors. Science. 2003;301(5638):1374-1377.

6. Avrameas S, Selmi C. Natural autoantibodies in the physiology and pathophysiology of the immune system. J Autoimmun. 2013;41:46-49.

7. Meffre E. The establishment of early B cell tolerance in humans: lessons from primary immunodeficiency diseases. Ann N Y Acad Sci. 2011;1246:1-10.

8. Pala F, et al. Lentiviral-mediated gene therapy restores B cell tolerance in Wiskott-
Aldrich syndrome patients. J Clin Invest. 125(10):3941-3951.

9. Catucci M, Castiello MC, Pala F, Bosticardo M, Villa A. Autoimmunity in Wiskott-Aldrich syndrome: an unsolved enigma. Front Immunol. 2012;3:209.

10. Aiuti A, et al. Lentiviral hematopoietic stem cell gene therapy in patients with Wiskott-Aldrich syndrome. Science. 2013;341(6148):1233151.

11. Hacein-Bey Abina S, et al. Outcomes following gene therapy in patients with severe WiskottAldrich syndrome. JAMA. 2015;313(15):1550-1563.

12. Sauer AV, Morbach H, Brigida I, Ng YS, Aiuti A, Meffre E. Defective B cell tolerance in adenosine deaminase deficiency is corrected by gene therapy. JClin Invest. 2012;122(6):2141-2152.

13. Castiello MC, et al. B cell reconstitution after lentiviral vector-mediated gene therapy in patients with Wiskott-Aldrich syndrome. J Allergy Clin Immunol. 2015;136(3):692-702.e2.

14. Kinnunen T, et al. Accumulation of peripheral autoreactive B cells in the absence of functional human regulatory T cells. Blood. 2013;121(9):1595-1603.

15. Iikuni N, Lourenco EV, Hahn BH, La Cava A. Cutting edge: Regulatory T cells directly suppress B cells in systemic lupus erythematosus. J Immunol. 2009;183(3):1518-1522.

16. Menard L, et al. Signaling lymphocytic activation molecule (SLAM)/SLAM-associated protein pathway regulates human B cell tolerance. J Allergy Clin Immunol. 2014;133(4):1149-1161.

17. Rathmell JC, et al. CD95 (Fas)-dependent elimination of self-reactive B cells upon interaction with $\mathrm{CD}^{+} \mathrm{T}$ cells. Nature. 1995;376(6536):181-184. 